\title{
Longitudinal Chromatic Aberration in Patients Implanted With Trifocal Diffractive Hydrophobic IOLs
}

Maria Vinas, PhD; Ana Maria Gonzalez-Ramos, MSc; Sara Aissati, MSc; Nuria Garzón, PhD; Francisco Poyales, MD; Carlos Dorronsoro, PhD; Susana Marcos, PhD

\section{ABSTRACT}

PURPOSE: To measure the in vivo longitudinal chromatic aberration (LCA) from the chromatic difference of focus ( 480 to $700 \mathrm{~nm}$ ) using psychophysical methods in patients bilaterally implanted with a hydrophobic trifocal intraocular lens (IOL).

METHODS: Psychophysical best focus was measured in both eyes at different wavelengths ( 480 to $700 \mathrm{~nm})$ and at three different viewing distances $(0.00,+1.75$, and +3.50 diopters [D]) using a custom-developed polychromatic adaptive optics set-up provided with a supercontinuum laser, a Hartmann-Shack wavefront sensor, a deformable mirror, a motorized Badal system, a pupil monitoring system, and a psychophysical channel with monochromatically illuminated stimuli. Measurements were performed on 10 patients (20 eyes) bilaterally implanted with hydrophobic trifocal diffractive IOLs (FineVisionHP POD F GF; PhysIOL). LCA was com- puted from the chromatic difference of focus curves as the difference between 480 and $700 \mathrm{~nm}$ at near, intermediate, and far.

RESULTS: The LCA from psychophysical measurements was significantly higher for far vision $[0.99 \pm 0.06$ diopters [D]), than for intermediate $(0.67 \pm 0.10 \mathrm{D})$ and near ( $0.23 \pm 0.08 \mathrm{D})$ vision (one-way analysis of variance, $P<.05$ )

CONCLUSIONS: LCA for far vision was significantly higher than for intermediate and near vision in hydrophobic trifocal diffractive IOLs, in agreement with a previous study with the same optical design but hydrophilic material IOLs. The LCA for the hydrophobic IOL is slightly higher than for the hydrophilic IOL at far. Different combinations of refractive and diffractive LCA will allow optimizing IOL designs to improve polychromatic image quality.

\section{[J Refract Surg. 20XX;X(X):XX-XX.]}

I ne natural world, the retinal image quality is affected by monochromatic and chromatic aberrations of the ocular optics and by interactions between them. Chromatic defocus results from the fact that the refractive index of the ocular media depends on the wavelength, ${ }^{1-3}$ and is such that the blue wavelengths focus in front of the retina and the red wavelengths behind it; the difference is known as longitudinal chromatic aberration (LCA).,

When an intraocular lens (IOL) replaces the natural crystalline lens of the eye, LCA changes according to the Abbe number of the IOL material. Various studies report the LCA of lenses with different materials, both on bench and once implanted in the eye. ${ }^{6-13}$ For example, the psy- chophysical LCA (480 to $700 \mathrm{~nm}$ ) in phakic eyes has been reported to be 1.52 diopters (D), ${ }^{5}$ whereas LCA was $1.37 \pm 0.08 \mathrm{D}$ in patients implanted with hydrophobic monofocal IOLs (FineVision hydrophobic raw material) and $1.21 \pm 0.08 \mathrm{D}$ in patients implanted with hydrophilic monofocal IOLs (FineVision hydrophilic raw material). ${ }^{14}$

Besides the material, the design of the $\mathrm{IOL}^{15}$ also influences the chromatic aberrations of IOLs. Therefore, the optical performance of the pseudophakic eye in polychromatic light will be determined by both the IOL material and the IOL design, which is especially relevant in diffractive designs. ${ }^{16-19}$ Using diffractive optics, it is possible to alter $^{20,21}$ and even change the sign of the chromatic aberra-

From the Instituto de Óptica, Consejo Superior de Investigaciones Científicas, Madrid, Spain (MV, AMG-R, SA, CD, SM); and Miranza IOA Madrid, Madrid, Spain (NG, FP).

Submitted: March 25, 2020; Accepted: September 28, 2020

Supported by Spanish Government Grant No. FIS 2017-84753 to Dr. Marcos, and FPU Predoctoral Fellowship No. FPU16/01944 to Ms. Aissati.

The authors have no financial or proprietary interest in the materials presented herein.

The authors thank PhysIOL for providing the intraocular lenses.

Correspondence: Maria Vinas, PhD, Instituto de Óptica. Consejo Superior de Investigaciones Científicas. Serrano, 121. 28006 Madrid. Spain. Email: maria.vinas@io.cfmac.csic.es 
tion induced by the lens, at least in several foci. ${ }^{18,22}$ Shifts in the position of the focus peaks of diffractive bifocal lenses with wavelength have also been strategically used to "fill in" intermediate distances and mimic an extended depth-of-focus in polychromatic light.

Millán et $\mathrm{al}^{23}$ evaluated, theoretically and on bench, the LCA and through-focus energy efficiency of four bifocal IOLs of apodized and non-apodized designs with different add powers and materials (Tecnis ZKB00 \& ZMA00 by Johnson \& Johnson and ReSTOR SN6AD3 and SV25T0 by Alcon Laboratories, Inc, respectively). They found that the chromatic defocus in bifocal IOLs due to the refractive base power is additive to the LCA of the ocular media for far vision and the achromatizing effect of diffractive bifocal IOLs compensates, in part, for the natural eye's LCA in near vision.

Using pseudophakic eye models, Ravikumar et $\mathrm{al}^{16}$ demonstrated that achromatization by a diffractive IOL may provide significant improvement in polychromatic retinal image quality, whereas the combination of LCA, multifocal design, apodization, and higher order aberrations can significantly affect the near-distance balance provided by a diffractive multifocal IOL.

The trifocal diffractive IOL hydrophilic FineVision POD $\mathrm{F}$ lens (PhysIOL), which has been available in many markets since 2012, combines two bifocal diffractive patterns (one for far and near vision, and the other for far and intermediate vision) designed to concentrate light into near $(+3.50 \mathrm{D})$, intermediate $(+1.75$ D), and distant foci. ${ }^{21,24}$ The apodized design of the FineVision trifocal technology benefits the far focus against near/intermediate focus for larger pupils. The energy balance is, as expected, wavelength-dependent, due to variations of diffraction efficiency with wavelength. On-bench studies ${ }^{18,25-28}$ compared the optical performance of diffractive multifocal IOLs with visible and near infrared light, showing a bias in optical performance of the IOL toward far focus for near infrared illumination.

Loicq et $\mathrm{al}^{22}$ published a comprehensive on-bench study comparing the through-focus modulation of nine different lenses in red, green, and blue wavelengths, and confirmed a compensatory effect between refractive and diffractive contributions for certain foci in diffractive lenses, the actual magnitude depending on material and design.

We recently presented the LCA measured in vivo in 20 eyes implanted with hydrophilic trifocal diffractive IOLs (FineVision POD F; PhysIOL) at three different viewing distances $(0.00,+1.75$, and $+3.50 \mathrm{D})$ in the spectral range of 480 to $700 \mathrm{~nm} .{ }^{29}$ We found that subjective LCA was higher for far $(0.82 \pm 0.05 \mathrm{D})$ than for intermediate $(0.27$ $\pm 0.15 \mathrm{D})$ and near $(0.15 \pm 0.15 \mathrm{D})$ vision.
A new trifocal lens design has been recently introduced by PhysIOL (the FineVisionHP POD F GF), with a similar topographic diffractive profile to the FineVision POD F design, but using a hydrophobic material. This gives us the unique opportunity to test in vivo the impact of the lens material alone on the chromatic performance of a diffractive trifocal IOL, at different foci.

In this study, we present the first in vivo measurements of LCA in patients bilaterally implanted with hydrophobic multifocal diffractive multifocal IOLs (FineVisionHP POD F GF) in the visible range (480 to $700 \mathrm{~nm}$ ), which will provide insights on the influence of material and multifocal IOL design on the LCA of pseudophakic patients, with a direct impact on the optical and visual performance with these lenses.

\section{PATIENTS AND METHODS}

LCA was obtained from psychophysical measurements of best focus at different wavelengths and viewing distances in 10 patients bilaterally implanted with a hydrophobic trifocal diffractive IOL (FineVisionHP POD F GF) in each eye. Measurements were performed using a custom-developed polychromatic adaptive optics system.

The instrument and protocols are similar to those described in an earlier publication. ${ }^{29}$ The cohort in the earlier publication were 10 patients (20 eyes) implanted with the hydophilic trifocal diffractive IOL. The current cohort is a new cohort of patients (FineVisionHP POD F).

\section{IOLS AND PATIENTS}

The patients enrolled in this study were bilaterally implanted with a hydrophobic multifocal diffractive IOL (FineVisionHP POD F GF), which is an aspheric diffractive IOL made with a hydrophobic glisteningfree material (Abbe number $=41.91$ ). The IOL design has been described previously. ${ }^{29,30}$ In brief, it combines two bifocal diffractive structures, one for far and near vision and the other for far and intermediate vision, to provide three useful focal distances: $0.00 \mathrm{D}$ for far vision, $+1.75 \mathrm{D}$ addition for intermediate vision, and $+3.50 \mathrm{D}$ addition for near vision.

Ten patients (20 eyes) participated in the current study (mean age: $64.56 \pm 3.52$ years, range: 53 to 71 years) after bilateral implantation of a hydrophobic diffractive multifocal IOL. Patients received a complete ophthalmic evaluation prior to enrollment in the study and surgery at Miranza IOA (Madrid, Spain). All participants were acquainted with the nature and possible consequences of the study and provided written informed consent. All protocols met the tenets of the Dec- 


\begin{tabular}{|c|c|c|c|c|c|c|c|}
\hline \multicolumn{8}{|c|}{$\begin{array}{c}\text { TABLE } 1 \\
\text { 1-Month Postoperative Clinical Data }\end{array}$} \\
\hline Patient/Sex/Age (y) & Eye & IOL Power (D) & Sphere (D) & Cylinder (D) & Axis $\left({ }^{\circ}\right)$ & UDVA & CDVA \\
\hline \multirow[t]{2}{*}{$\mathrm{S} 1 / \mathrm{M} / 53$} & OD & 22.00 & 0.00 & 0.00 & - & $20 / 10$ & $20 / 10$ \\
\hline & OS & 22.00 & 0.00 & 0.00 & - & $20 / 20$ & $20 / 20$ \\
\hline \multirow[t]{2}{*}{$\mathrm{S} 2 / \mathrm{F} / 70$} & OD & 21.00 & -0.50 & 0.00 & - & $20 / 25$ & $20 / 20$ \\
\hline & OS & 21.00 & -0.50 & 0.00 & - & $20 / 25$ & $20 / 20$ \\
\hline \multirow[t]{2}{*}{ S3/F/70 } & $O D$ & 23.50 & +0.50 & -0.50 & 95 & $20 / 25$ & $20 / 20$ \\
\hline & OS & 24.00 & +0.50 & -0.75 & 85 & $20 / 25$ & $20 / 20$ \\
\hline \multirow[t]{2}{*}{$\mathrm{S} 4 / \mathrm{F} / 53$} & OD & 22.50 & 0.00 & 0.00 & - & $20 / 20$ & $20 / 20$ \\
\hline & OS & 22.50 & 0.00 & 0.00 & - & $20 / 20$ & $20 / 20$ \\
\hline \multirow[t]{2}{*}{ S5/F/69 } & OD & 22.00 & 0.00 & 0.00 & - & $20 / 10$ & $20 / 10$ \\
\hline & OS & 23.00 & 0.00 & 0.00 & - & $20 / 20$ & $20 / 20$ \\
\hline \multirow[t]{2}{*}{ S6/F/67 } & OD & 24.50 & -1.00 & 0.00 & - & $20 / 40$ & $20 / 20$ \\
\hline & OS & 24.00 & +0.25 & -0.75 & 55 & $20 / 50$ & $20 / 20$ \\
\hline \multirow[t]{2}{*}{ S7/F/71 } & OD & 23.00 & +1.25 & -1.00 & 95 & $20 / 20$ & $20 / 20$ \\
\hline & OS & 22.50 & 0.00 & 0.00 & - & $20 / 20$ & $20 / 20$ \\
\hline \multirow[t]{2}{*}{ S8/M/63 } & OD & 25.50 & +0.75 & 0.00 & - & $20 / 30$ & $20 / 20$ \\
\hline & OS & 25.00 & +0.75 & 0.00 & - & $20 / 25$ & $20 / 20$ \\
\hline \multirow[t]{2}{*}{ S9/F/65 } & OD & 24.50 & 0.00 & 0.00 & - & $20 / 20$ & $20 / 20$ \\
\hline & OS & 26.00 & +0.75 & -0.75 & 180 & $20 / 25$ & $20 / 20$ \\
\hline \multirow[t]{2}{*}{$\mathrm{S} 10 / \mathrm{F} / 71$} & OD & 22.50 & -1.00 & 0.00 & - & $20 / 32$ & $20 / 20$ \\
\hline & OS & 22.00 & -0.50 & 0.00 & - & $20 / 25$ & $20 / 20$ \\
\hline
\end{tabular}

laration of Helsinki and were approved by the Spanish National Research Council Bioethical Committee.

The inclusion criteria were similar to those in our previous study, ${ }^{29}$ the most relevant being good general health, IOL power between 16.00 and 26.00 $\mathrm{D}$, and postoperative corrected distance visual acuity (CDVA) better than 0.7 decimal (20/30 Snellen or 0.16 logMAR). The same surgeon (FP) performed the surgery in both eyes of all patients, with a time difference of less than 3 days between surgeries, following the same surgical procedure described previously. ${ }^{29}$

Table 1 summarizes the patient profile, multifocal IOL characteristics, and 1-month postoperative data for all 10 patients in the current study.

\section{POLYCHROMATIC AdAPTIVE OPTICS SYSTEM}

A custom-developed 8-channel polychromatic adaptive optics system at the Visual Optics and Biophotonics Laboratory (Instituto de Óptica, Consejo Superior de Investigaciones Científicas), described in detail in previous publications, ${ }^{5,31-34}$ was used to perform psychophysical settings of best focus at differ- ent wavelengths while controlling the aberrations of the system. The components of the set-up specifically used in these measurements were: (1) a supercontinuum laser source in combination with a dual acoustooptic tunable filter module to automatically select the desired wavelengths in visible light (480 to $700 \mathrm{~nm}$ ), where the laser power at the corneal plane ranged between 0.5 and $50 \mu \mathrm{W}$; (2) a digital micro-mirror device placed in a conjugate retinal plane, and used to display visual stimuli (1.62 degree field) monochromatically illuminated with light coming from the supercontinuum laser source; (3) a Badal optometer used to correct the spherical refractive error of the patients and the best focus settings; and (4) a pupil monitoring camera conjugated to the eye's pupil to facilitate centration and control of aberrations.

\section{PSYCHOPHYSICAL BEST FOCUS AT DIFFERENT WAVELENGTHS AND DISTANCES}

Patients adjusted their best subjective focus using the Badal system while viewing the stimulus illuminated with five different wavelengths in visible light (480, 532, 555, 650, and $700 \mathrm{~nm}$ ). Best subjective focus was 
set remotely by the patient moving the motorized stage while viewing a Maltese cross as a fixation target, starting from an initial positive blur. Patients performed a trial before the experiment to become familiar with the test, and afterward repeated each wavelength condition four times. The focus setting for the stimulus illuminated with a 555-nm wavelength was set as zero.

All measurements were performed under mydriasis (tropicamide 1\%; two drops 30 minutes before the test start, and one drop every 1 hour) of both eyes. Measurements were performed using a 4-mm diameter pupil (achieved by placing an artificial pupil in a plane conjugate to the natural pupil), with adaptive opticscorrected aberrations of the optical system. The eye's pupil was aligned to the optical axis of the instrument, and the patient's head was stabilized using a dental impression on a bite bar. The patient's spherical refractive error was corrected with a Badal system.

The different wavelengths were presented randomly. Measurements were performed at three different viewing distances simulated with the Badal system: $0.00 \mathrm{D}$ for far vision, +1.75 D for intermediate vision, and $+3.50 \mathrm{D}$ for near vision. Each measurement session lasted approximately 3 hours.

\section{DATA ANALYSIS}

The best subjective focus at each wavelength was directly obtained from the automatic readings of the Badal optometer. Chromatic difference of focus curves were obtained from the best focus data at each wavelength. The LCA was obtained from the linear fittings to those curves.

Statistical analysis was performed with SPSS software (version 24.0; IBM Corporation) to test differences in the estimated LCA across experiments and conditions. A paired samples $t$ test was performed to analyze specific differences between conditions ( $\mathrm{n}=$ 10 , alpha $=0.05$, power $=0.80$ ).

\section{RESULTS}

Figure A (available in the online version of this article) shows the refractive outcomes.

\section{CHROMATIC DIFFERENCE OF FOCUS FROM PSYCHOPHYSICAL MEASUREMENTS}

Figure B (available in the online version of this article) shows the chromatic difference of focus curves obtained from the focus settings for all patients and distances (far: green; intermediate: orange; near: purple) for both eyes (right eye = left column; left eye = right column). Lines represent linear fitting curves to the data. Results show similar trends across patients for the different distances. On average, the regression lines show higher slopes for far $(0.005 \pm 0.0005 \mathrm{D} / \mathrm{nm})$ than for intermediate $(0.003 \pm 0.0006 \mathrm{D} / \mathrm{nm})$ and near $(0.001 \pm 0.0007 \mathrm{D} / \mathrm{nm})$ distances. The standard deviations are higher for near than for intermediate and far distances. We did not find statistical differences in the slopes between right and left eyes: $0.005 \pm 0.0005$ $\mathrm{D} / \mathrm{nm}$ (right eye) and $0.004 \pm 0.0006 \mathrm{D} / \mathrm{nm}$ (left eye) $(P=.46)$ for far; $0.003 \pm 0.0006 \mathrm{D} / \mathrm{nm}$ (right eye) and $0.003 \pm 0.0007 \mathrm{D} / \mathrm{nm}$ (right eye) $(P=.33)$ for intermediate; and $0.001 \pm 0.0008 \mathrm{D} / \mathrm{nm}$ (right eye) and $0.001 \pm$ $0.0007 \mathrm{D} / \mathrm{mm}$ (left eye) $(P=.05)$ for near.

Figure 1 shows the LCA from psychophysical measurements for all patients in both eyes and all tested distances (far: green bars; intermediate: orange bars; near: purple bars) in the visible range (480 to $700 \mathrm{~nm}$ ). Results show similar trends for all patients and both eyes, with higher LCA for far than for intermediate and near vision.

Figure 2 shows the average LCA from psychophysical measurements for far (green), intermediate (orange), and near (purple) vision in the measured visible range (480 to $700 \mathrm{~nm}$ ), across patients. The LCA from psychophysical measurements was significantly higher for far vision $(0.99 \pm 0.06 \mathrm{D})$ than for intermediate $(0.67 \pm 0.10 \mathrm{D})$ and near $(0.23 \pm 0.08 \mathrm{D})$ vision (one-way analysis of variance, $P<.05$ ).

\section{DISCUSSION}

The optical performance of the pseudophakic eye in polychromatic light is determined by both the material and design of the IOL. This is especially relevant in diffractive designs, such as trifocal diffractive IOLs, aiming at generating an intermediate focal point in the IOL optic to improve the optical range. In a previous study, we measured the in vivo subjective LCA of 20 eyes bilaterally implanted with hydrophilic trifocal diffractive IOLs (FineVision POD F) for the different working distances: $0.00 \mathrm{D}$ for far, +1.75 D for intermediate, and +3.50 D for near distances. The LCA from psychophysical measurements was significantly higher for far $(0.82 \pm 0.05 \mathrm{D})$ than for intermediate $(0.27 \pm 0.15 \mathrm{D})$ and near $(0.15 \pm 0.15 \mathrm{D})$ vision. In the current study, LCA of 20 eyes implanted with same design but hydrophobic material (FineVisionHP POD F GF) was obtained from in vivo psychophysical measurements (480 to $700 \mathrm{~nm}$ ). Similarly, to the hydrophilic design, LCA for far vision was significantly higher than for intermediate and near vision (far: $0.99 \pm 0.06 \mathrm{D}$; intermediate: $0.67 \pm 0.10 \mathrm{D}$; near: $0.23 \pm 0.08 \mathrm{D}$ ), and, on average, LCA with the hydrophobic IOL was higher than with the hydrophilic IOL for all distances.

Diffractive multifocal IOLs are typically a hybrid refractive-diffractive design, where light energy is split between different foci. In most designs, the far foci receive light that appears to be purely refracted (0 diffraction order), whereas the other foci (inter- 


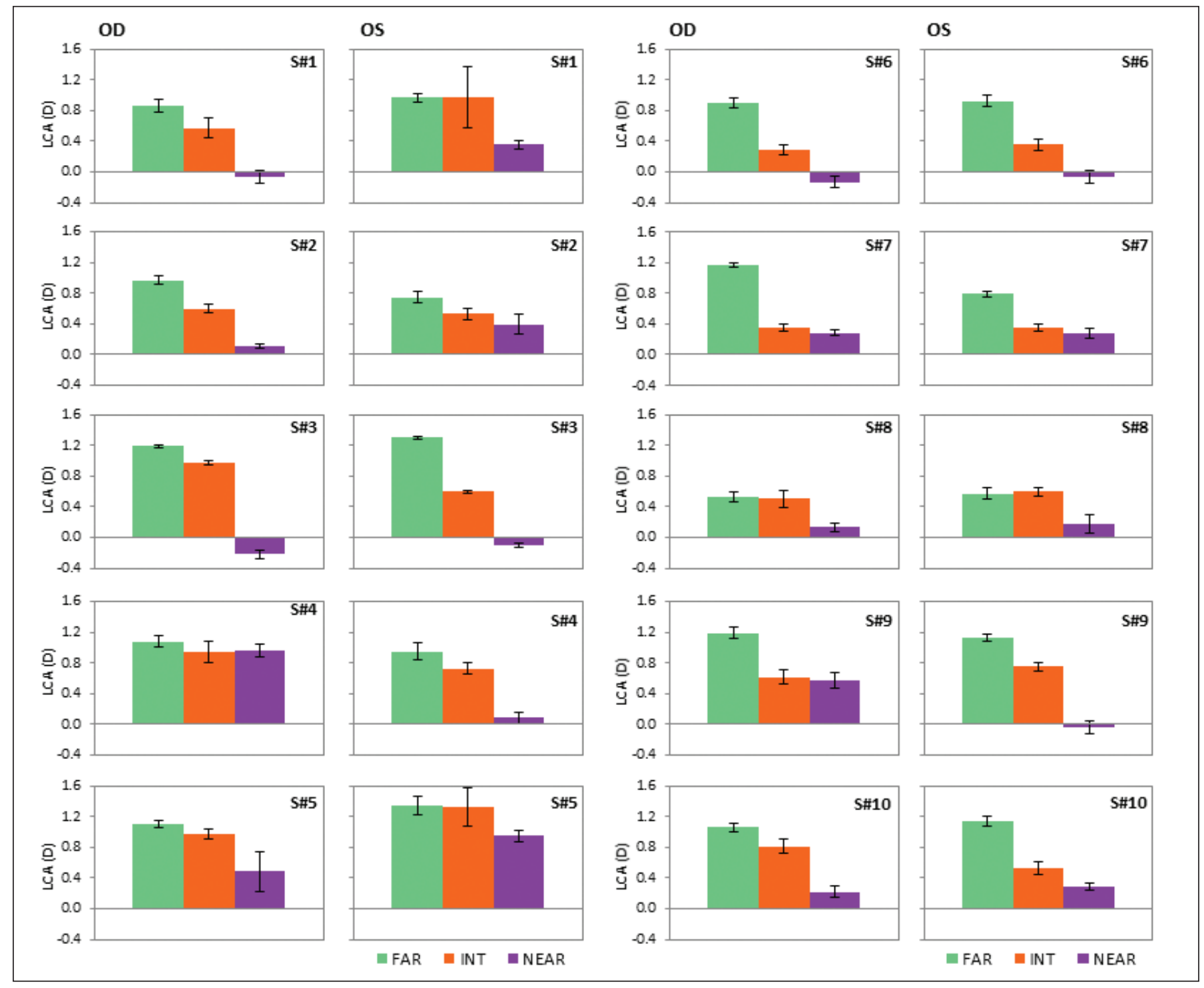

Figure 1. Longitudinal chromatic aberration (LCA) obtained from linear fitting of the chromatic difference of focus curves ( 480 to $700 \mathrm{~nm}$ ) for all patients, and for each measured distance (far: green bars; intermediate: orange bars; near: purple bars). Error bars indicate inter-patient variability. $\mathrm{D}=$ diopters; $\mathrm{OD}=$ right eye; $\mathrm{OS}=$ left eye

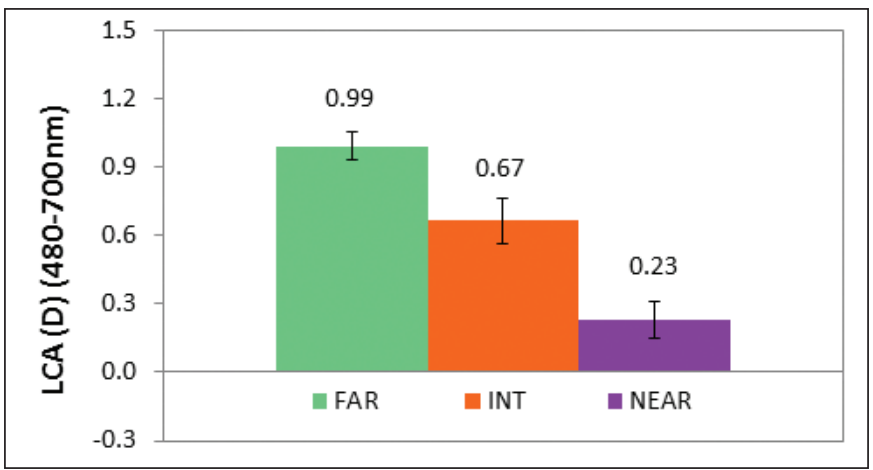

Figure 2. Average longitudinal chromatic aberration (LCA) from psychophysical measurements for all measured distances (far: green bars; intermediate: orange bars; near: purple bars). Error bars indicate inter-patient variability. $\mathrm{D}=$ diopters mediate and near) are obtained through a combination of diffracted light (first and second order of diffraction). Refractive or diffractive focalization leads to opposite signs of LCA, thus allowing modulation of the chromatic aberration of the eye at different distances. The decrease of the LCA at intermediate distance in patients implanted with the FineVision trifocal IOL, for both materials, is also supported by recent on-bench work by Loicq et al. ${ }^{22}$ They found that for far vision there is a refractive dominant process, whereas for near the trend is reversed and there is a diffractive dominant process. The LCA (of the IOL alone) was almost fully cancelled at intermediate distance and its sign was reversed at near distance. 
This agrees well with our findings of reduction and almost full cancelation at intermediate and near distance in eyes implanted with the IOL.

At every distance, we found a consistently higher LCA value in patients implanted with the hydrophobic IOL than with the hydrophilic IOL. This is consistent with a previous study where patients were bilaterally implanted with monofocal IOLs of the same design, but with hydrophobic material in one eye and hydrophilic material in the contralateral eye. ${ }^{13}$ In that study, there was a constant offset of $0.15 \mathrm{D}$ between the LCA measured in vivo in eyes implanted with monofocal IOLs of both materials, which compares with the offset in the LCA found between the hydrophobic and hydrophilic trifocal IOL at far (0.17 D). However, the magnitude of the LCA in patients implanted with monofocal IOL designs was higher $(1.37 \pm 0.08 \mathrm{D}$ for the hydrophobic and 1.2.1 $\pm 0.08 \mathrm{D}$ for the hydrophilic) than with the corresponding trifocal designs.

The ability to measure in vivo LCA in monofocal and multifocal IOLs with different materials and designs allows quantifying retinal image quality in polychromatic pseudophakic eyes. Understanding the interactions between monochromatic and chromatic aberrations of the eye and the different multifocal diffractive design improves the resulting polychromatic retinal quality and simultaneous vision quality, therefore opening the path to real custom designs.

\section{AUTHOR CONTRIBUTIONS}

Study concept and design (MV, SM); patients recruitment and surgery (NG, FP); data collection (MV, AMG-R, SA); analysis and interpretation of data (MV, $\mathrm{SM}$ ); writing the manuscript (MV, SM); critical revision of the manuscript (MV, AMG-R, SA, NG, FP, CD, SM)

\section{REFERENCES}

1. Thibos LN, Bradley A, Zhang XX. Effect of ocular chromatic aberration on monocular visual performance. Optom Vis Sci. 1991;68(8):599-607. doi:10.1097/00006324-199108000-00005

2. Graef K, Schaeffel F. Control of accommodation by longitudinal chromatic aberration and blue cones. J Vis. 2012;12(1):14. doi:10.1167/12.1.14

3. Charman WN. Optics of the Human Eye. CRC Press; 1991.

4. Bedford RE, Wyszecki G. Axial chromatic aberration of the human eye. J Opt Soc Am. 1957;47(6):564-565. doi:10.1364/ JOSA.47.0564_1

5. Vinas M, Dorronsoro C, Cortes D, Pascual D, Marcos S. Longitudinal chromatic aberration of the human eye in the visible and near infrared from wavefront sensing, double-pass and psychophysics. Biomed Opt Express. 2015;6(3):948-962. doi:10.1364/ BOE.6.000948

6. Nakajima M, Hiraoka T, Yamamoto $\mathrm{T}$, et al. Differences of longitudinal chromatic aberration (LCA) between eyes with intraocular lenses from different manufacturers. PLoS One. 2016;11(6):e0156227. doi:10.1371/journal.pone.0156227
7. Zhao H, Mainster MA. The effect of chromatic dispersion on pseudophakic optical performance. Br J Ophthalmol. 2007;91(9):12251229. doi:10.1136/bjo.2007.118745

8. Nagata T, Kubota S, Watanabe I, Aoshima S. [Chromatic aberration in pseudophakic eyes]. Nippon Ganka Gakkai Zasshi. 1999;103(3):237-242.

9. Pérez-Merino P, Dorronsoro C, Llorente L, Durán S, JiménezAlfaro I, Marcos S. In vivo chromatic aberration in eyes implanted with intraocular lenses. Invest Ophthalmol Vis Sci. 2013;54(4):2654-2661. doi:10.1167/iovs.13-11912

10. Siedlecki D, Józwik A, Zajac M, Hill-Bator A, Turno-Krecicka A. In vivo longitudinal chromatic aberration of pseudophakic eyes. Optom Vis Sci. 2014;91(2):240-246.

11. Negishi K, Ohnuma K, Hirayama N, Noda T; Policy-Based Medical Services Network Study Group for Intraocular Lens and Refractive Surgery. Effect of chromatic aberration on contrast sensitivity in pseudophakic eyes. Arch Ophthalmol. 2001;119(8):1154-1158. doi:10.1001/archopht.119.8.1154

12. Bobier CW, Sivak JG. Chromoretinoscopy. Vision Res. 1978;18(3):247-250. doi:10.1016/0042-6989(78)90158-X

13. Marcos S, Romero M, Benedí-García C, et al. Interaction of monochromatic and chromatic aberrations in pseudophakic patients. J Refract Surg. 2020;36(4):230-238. doi:10.3928/1081 597X-20200303-01

14. Vinas M, Dorronsoro C, Garzón N, Poyales F, Marcos S. In vivo subjective and objective longitudinal chromatic aberration after bilateral implantation of the same design of hydrophobic and hydrophilic intraocular lenses. J Cataract Refract Surg. 2015;41(10):2115-2124. doi:10.1016/j.jcrs.2015.11.009

15. Charman WN. Developments in the correction of presbyopia II: surgical approaches. Ophthalmic \& Physiological Optics. 2014;34(4):397-426.

16. Ravikumar S, Bradley A, Thibos LN. Chromatic aberration and polychromatic image quality with diffractive multifocal intraocular lenses. J Cataract Refract Surg. 2014;40(7):1192-1204. doi:10.1016/j.jcrs.2013.11.035

17. Castignoles F, Flury M, Lepine T. Comparison of the efficiency, MTF and chromatic properties of four diffractive bifocal intraocular lens designs. Opt Express. 2010;18(5):5245-5256. doi:10.1364/OE.18.005245

18. Vega F, Millán MS, Vila-Terricabras N, Alba-Bueno F. Visible versus near-infrared optical performance of diffractive multifocal intraocular lenses. Invest Ophthalmol Vis Sci. 2015;56(12):7345-7351. doi:10.1167/iovs.15-17664

19. Simpson MJ. Diffractive multifocal intraocular lens image quality. Appl Opt. 1992;31(19):3621-3626. doi:10.1364/AO.31.003621

20. Davison JA, Simpson MJ. History and development of the apodized diffractive intraocular lens. J Cataract Refract Surg. 2006;32(5):849-858. doi:10.1016/j.jcrs.2006.02.006

21. Gatinel D, Houbrechts Y. Comparison of bifocal and trifocal diffractive and refractive intraocular lenses using an optical bench. J Cataract Refract Surg. 2013;39(7):1093-1099. doi:10.1016/j.jcrs.2013.01.048

22. Loicq J, Willet N, Gatinel D. Topography and longitudinal chromatic aberration characterizations of refractive-diffractive multifocal intraocular lenses. J Cataract Refract Surg. 2019;45(11):1650-1659. doi:10.1016/j.jcrs.2019.06.002

23. Millán MS, Vega F, Ríos-López I. Polychromatic image performance of diffractive bifocal intraocular lenses: longitudinal chromatic aberration and energy efficiency. Invest Ophthalmol Vis Sci. 2016;57(4):2021-2028. doi:10.1167/iovs.15-18861

24. Gatinel D, Pagnoulle C, Houbrechts Y, Gobin L. Design and qualification of a diffractive trifocal optical profile for intra- 
ocular lenses. J Cataract Refract Surg. 2011;37(11):2060-2067. doi:10.1016/j.jcrs.2011.05.047

25. Pepose JS, Wang D, Altmann GE. Comparison of through-focus image sharpness across five presbyopia-correcting intraocular lenses. Am J Ophthalmol. 2012;154(1):20-28.e1. doi:10.1016/j. ajo.2012.01.013

26. Kim MJ, Zheleznyak L, Macrae S, Tchah H, Yoon G. Objective evaluation of through-focus optical performance of presbyopiacorrecting intraocular lenses using an optical bench system. J Cataract Refract Surg. 2011;37(7):1305-1312. doi:10.1016/j. jcrs.2011.03.033

27. Maxwell WA, Lane SS, Zhou F. Performance of presbyopia-correcting intraocular lenses in distance optical bench tests. J Cataract Refract Surg. 2009;35(1):166-171. doi:10.1016/j.jcrs.2008.10.026

28. Vega F, Alba-Bueno F, Millán MS. Energy distribution between distance and near images in apodized diffractive multifocal intraocular lenses. Invest Ophthalmol Vis Sci. 2011;52(8):56955701. doi:10.1167/iovs.10-7123

29. Vinas M, Gonzalez-Ramos A, Dorronsoro C, et al. In vivo measurement of longitudinal chromatic aberration in patients im- planted with trifocal diffractive intraocular lenses. J Refract Surg. 2017;33(11):736-742. doi:10.3928/1081597X-20170814-01

30. Gatinel D, Pagnoulle C, Houbrechts Y, Gobin L. Design and qualification of a diffractive trifocal optical profile for intraocular lenses. J Cataract Refract Surg. 2011;37:2060-2067. doi:10.1016/j.jcrs.2011.05.047

31. Vinas M, Dorronsoro C, Gonzalez V, Cortes D, Radhakrishnan A, Marcos S. Testing vision with angular and radial multifocal designs using adaptive optics. Vision Res. 2017;132:85-96.

32. Vinas M, Dorronsoro C, Radhakrishnan A, et al. Comparison of vision through surface modulated and spatial light modulated multifocal optics. Biomed Opt Express. 2017;8(4):2055-2068. doi:10.1364/BOE.8.002055

33. Vinas M, Benedi-Garcia C, Aissati S, et al. Visual simulators replicate vision with multifocal lenses. Sci Rep. 2019;9(1):1539. doi:10.1038/s41598-019-38673-w

34. Vinas M, Aissati S, Romero M, et al. Preoperative simulation of postoperative multifocal vision. Biomed Opt Express. 2019;10(11):5801-5817. doi:10.1364/BOE.10.005801 


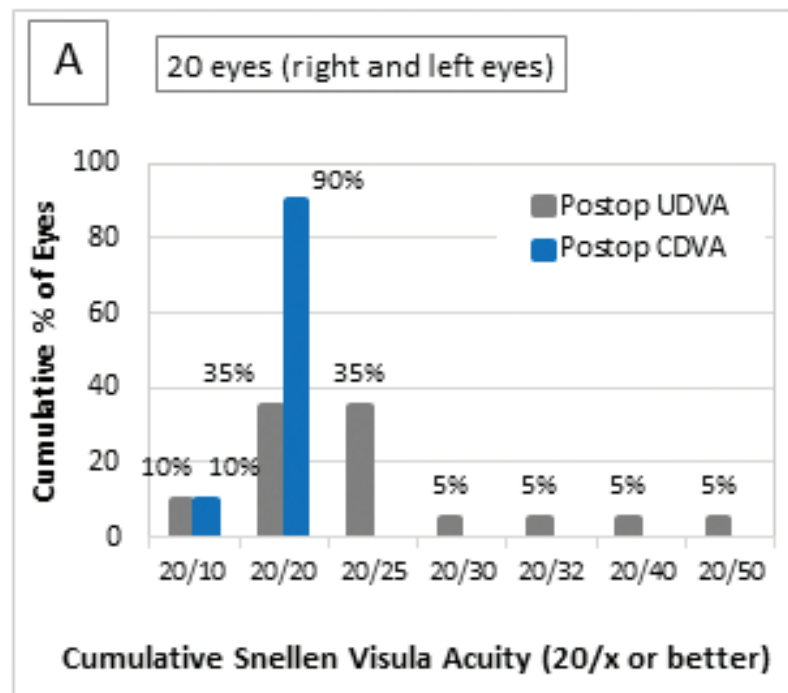

\section{Uncorrected Distance Visual Acuity}

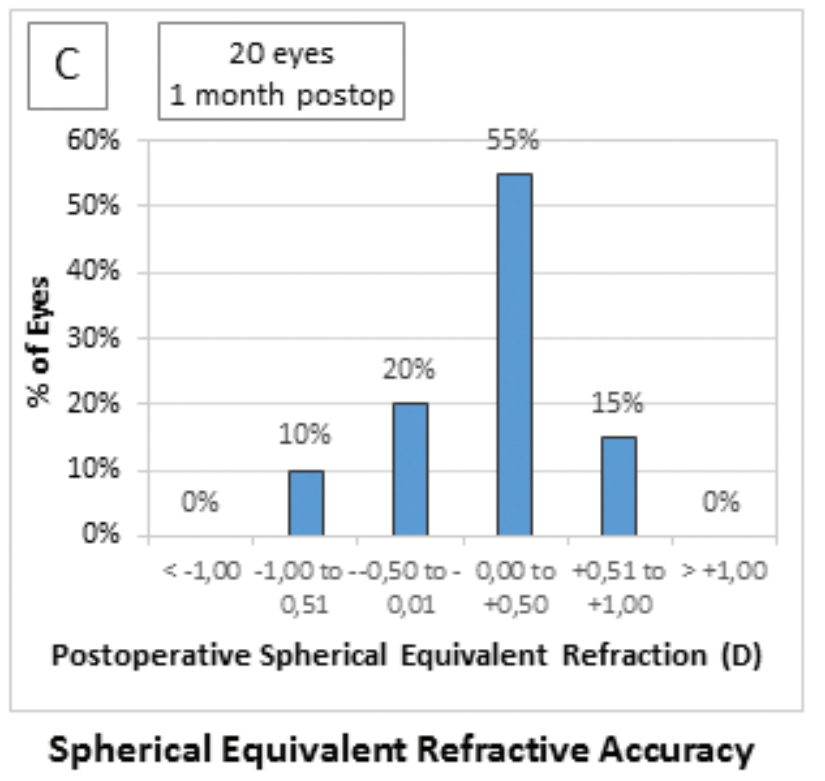

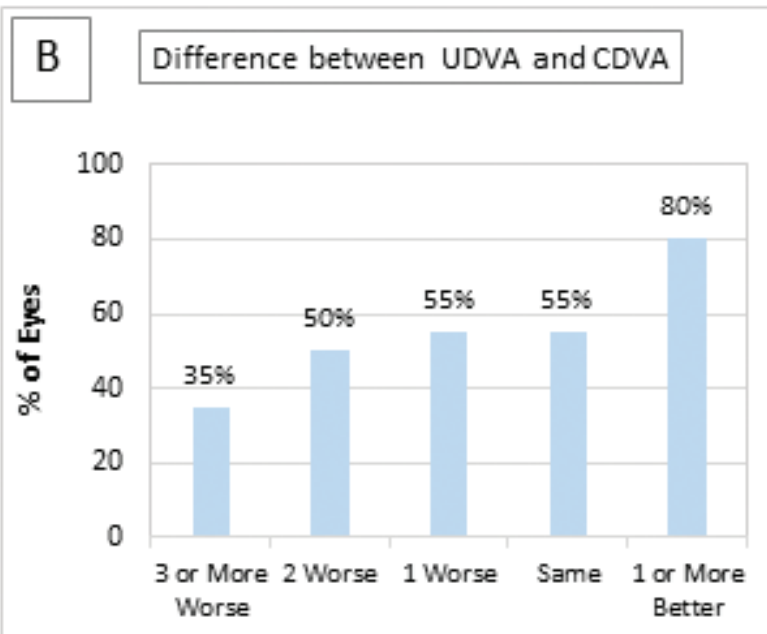

Difference between UDVA and CDVA (Snellen Lines)

\section{Uncorrected Distance Visual Acuity vs. Corrected Distance Visual Acuity}

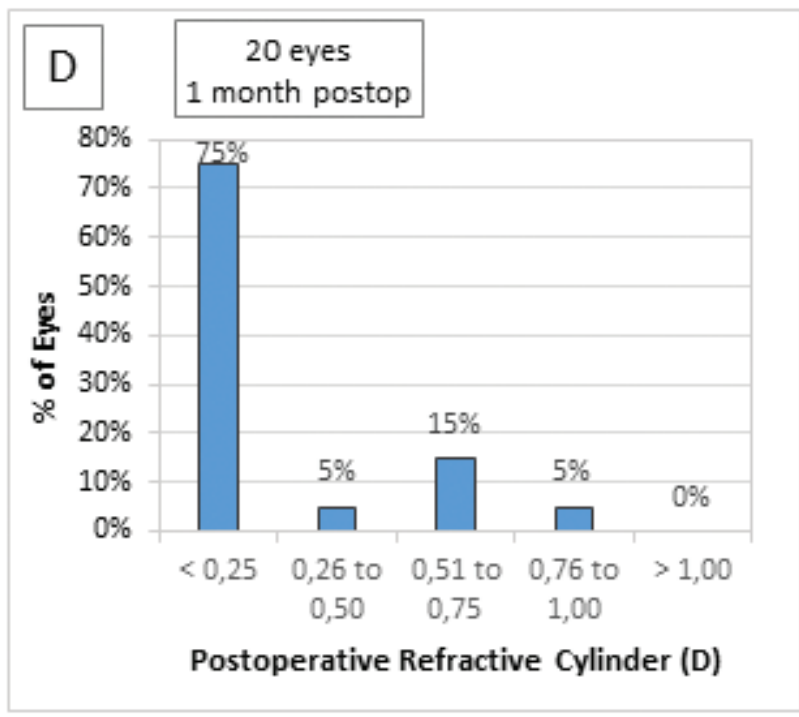

\section{Refractive cylinder}

Figure A. Refractive outcomes for intraocular lens-based procedures in the sample's study: (A) uncorrected distance visual acuity (UDVA); (B) UDVA vs corrected distance visual acuity (CDVA); (C) spherical equivalent refraction accuracy; and $(\mathrm{D})$ postoperative refractive cylinder. D = diopters 


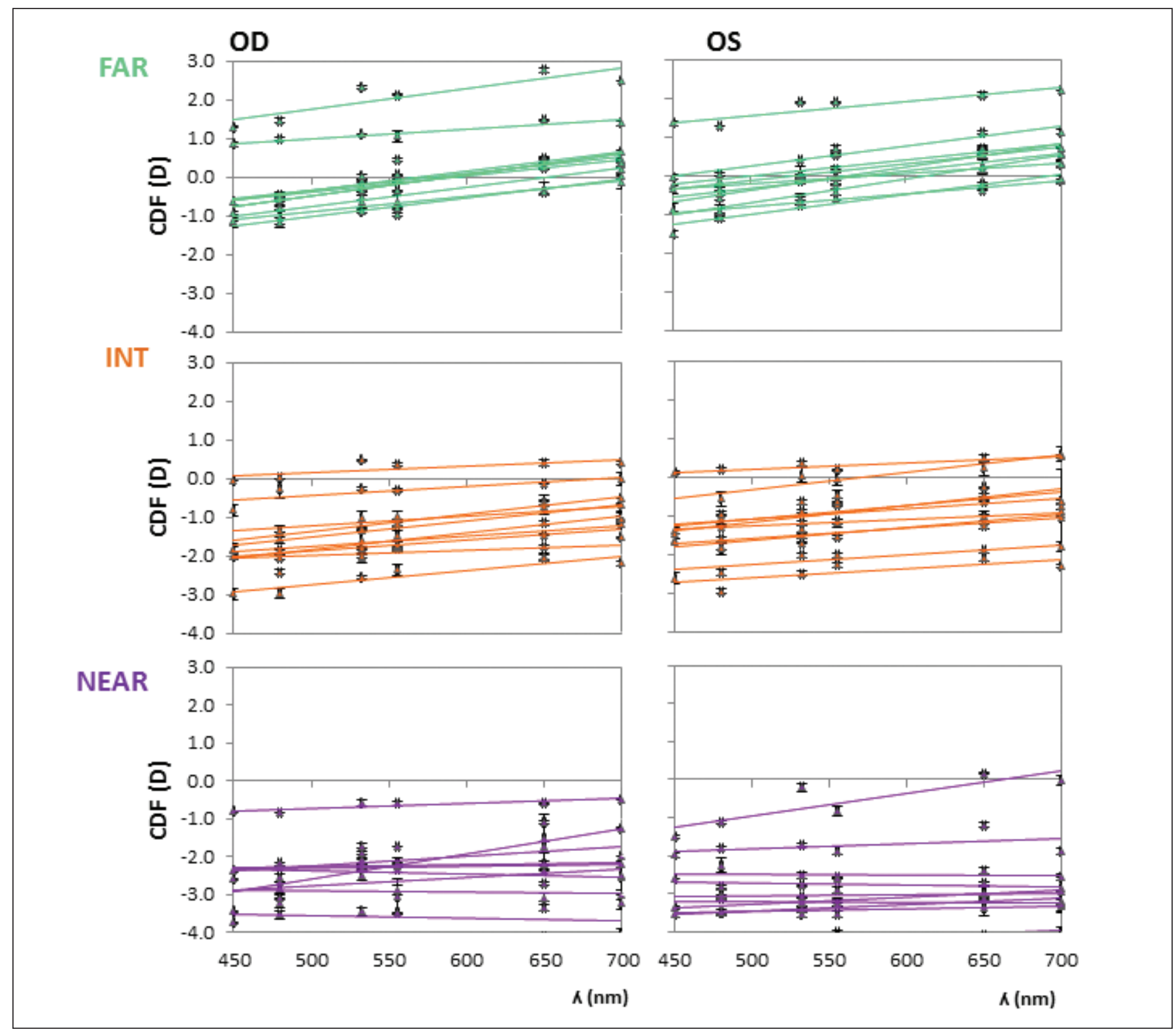

Figure B. Chromatic difference of focus (CDF). Psychophysical best focus chosen by each patient for each wavelength $(480,532,555,650$, and 700 $\mathrm{nm}$ ), distance (far: green; intermediate: orange; near: purple), and eye (right eye, left column; left eye, right column). Error bars indicate standard deviation for each measurement. $\mathrm{D}=$ diopters; $\mathrm{OD}$ = right eye; $\mathrm{OS}$ = left eye 